\title{
LINC01207 promotes prostate cancer progression by sponging miR-1182 to upregulate AKT3
}

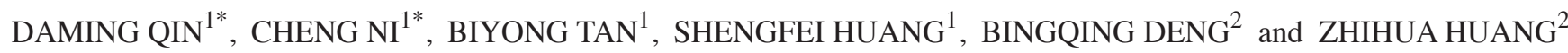 \\ Departments of ${ }^{1}$ Radiology and ${ }^{2}$ Ultrasonography, The Central Hospital of Enshi Tujia and Miao Autonomous Prefecture, \\ Enshi Clinical College of Wuhan University, Enshi, Hubei 445000, P.R. China
}

Received November 27, 2020; Accepted July 30, 2021

DOI: $10.3892 / \mathrm{ol} .2021 .13175$

\begin{abstract}
Prostate cancer (PC) is recognized as a common malignancy in male patients. Long non-coding RNA (lncRNA) has been implicated in the development of PC. Recently, long intergenic non-protein coding RNA 1207 (LINC01207) has been reported to regulate the carcinogenesis of multiple cancer types. However, its role in the progression of PC remains to be determined. The aim of the present study was to investigate the expression profile, clinicopathological implication and molecular mechanism of action of LINC01207 in the progression of PC. LINC01207 expression levels were compared between PC tumor and paired normal tissue samples from The Cancer Genome Atlas. The expression of LINC01207 was further analyzed in PC cell lines and a normal prostatic cell line. The role of LINC01207 in proliferation, migration and invasion of PC cells was examined using small interfering RNA-mediated silencing. Western blot analysis was used to investigate the changes in protein levels underlying the mechanism of action of LINC01207. The role of LINC01207 in tumorigenesis was evaluated in a xenograft model. LINC01207 was upregulated in PC tumor samples from TCGA data compared with paired normal tissue. LINC01207 expression was significantly increased in PC cells and tumor tissues compared with in normal prostate cells (RWPE1) and normal prostate tissues, respectively. Furthermore, LINC01207 silencing inhibited PC cell proliferation and colony formation and induced apoptosis. Mechanistic experiments showed that LINC01207 promoted carcinogenesis by sponging miR-1182 to regulate the protein levels of AKT3 in PC cell lines. Thus, the findings of the
\end{abstract}

Correspondence to: Dr Bingqing Deng or Dr Zhihua Huang, Department of Ultrasonography, The Central Hospital of Enshi Tujia and Miao Autonomous Prefecture, Enshi Clinical College of Wuhan University, 158 Wuyang Dadao, Enshi, Hubei 445000, P.R. China

E-mail: dbq208@163.com

E-mail: zhaoyuanli@pkuih.edu.cn

${ }^{*}$ Contributed equally

Key words: LINC01207, miR-1182, AKT3, prostate cancer, The Cancer Genome Atlas present study indicated that LINC01207 might play a role in the tumorigenesis of $\mathrm{PC}$ and may serve as a therapeutic target for PC treatment.

\section{Introduction}

Prostate cancer (PC) is a prevalent male cancer in European and American countries recognized as the second leading cause of cancer-associated mortality globally $(1,2)$. A previous study has detected novel molecular targets for PC therapy, however, the key initiating events regulating the pathogenesis and progression of PC remain elusive (3). The diagnosis of PC is mainly based on monitoring serum prostate-specific antigen (PSA) levels (4). Although the introduction of PSA testing facilitates early PC diagnosis, the clinical accuracy of PSA remains suboptimal to its lack of specificity and high false positive rate (5). Identifying novel biomarkers could improve the diagnosis accuracy in the early stages of PC, which may be beneficial for early intervention and for the prevention of progression to advanced stages of the disease (6).

Advances in whole-genome sequencing has facilitated the identification of non-coding RNA (ncRNA) in cancer, which has demonstrated that abnormal expression of ncRNA is associated with to PC progression (7). MicroRNA (miRNA/miR) is a type of endogenous ncRNA 21 nucleotides in length, which can bind to mRNA targets and regulate their expression at the post-transcriptional level (8). Increasing evidence suggests that several miRNA molecules can act as tumor suppressors or oncogenes in carcinogenesis $(9,10)$. Profiling the miRNA expression in different cancer types has identified abnormally expressed miRNA molecules which may serve as biomarkers for diagnosis and prognosis (11). Abnormal miRNA expression has also been observed in PC, and complex miRNA regulatory networks and modules have been proposed to regulate $\mathrm{PC}$ progression $(12,13)$. Aberrant miRNA expression frequently dysregulates the target genes implicated in carcinogenesis of PC $(14,15)$. Among them, miR-1182 was identified as a tumor suppressor and implicated in the tumorigenesis of different tumor types, such as bladder (16), ovarian (17), gastric (18) and PC (19). However, its regulatory mechanism of action in PC progression remains elusive.

The Akt serine/threonine kinase kinase family comprises three highly homologous members: AKT1 (PKB $\alpha)$, AKT2 $(\mathrm{PKB} \beta)$ and AKT3 (PKB $\gamma)(20)$. AKT3 is known to play role 
in carcinogenesis $(21,22)$. AKT3 is abnormally expressed in $\mathrm{PC}$, and its upregulation may promote PC cell survival and proliferation $(23,24)$. Small interfering RNA (siRNA)-mediated AKT3 silencing inhibits PC cell growth (25). Therefore, AKT3 signaling may play a vital role in PC tumorigenesis.

Located on chromosome 4q32, long intergenic non-protein coding RNA 1207 (LINC01207) consists of two introns and three exons (26-28). Recent studies have suggested that LINC01207 is highly expressed in lung adenocarcinoma and promotes cell proliferation and migration $(26,29)$. However, whether LINC01207 is involved in PC progression remains unclear.

The present study aimed to detect the role of LINC01207 in PC progression. The results demonstrated that LINC01207 was significantly upregulated in PC compared with normal prostate tissue samples. Mechanistic experiments demonstrated that LINC01207 played its oncogenic role by sustaining AKT3 levels in PC cells and sponging miR-1182. LINC01207 knockdown reduced AKT3 levels and induced apoptosis in PC cells. These findings identified a novel mechanism underlying the oncogenic role of LINC01207 by targeting the miR-1182/AKT3 axis in PC.

\section{Materials and methods}

Cell lines and cell culture. The human PC cell lines, PC-3, LNCaP, Du-145 and C4-2B, and the human normal prostate cell line, RWPE1, were purchased from American Type Culture Collection. These cell lines were cultured in DMEM (Gibco; Thermo Fisher Scientific, Inc.) containing 10\% FBS (Gibco; Thermo Fisher Scientific, Inc.) and $1 \%$ penicillin-streptomycin, and incubated in a humidified cell culture incubator at $37^{\circ} \mathrm{C}$ with $5 \% \mathrm{CO}_{2}$.

Cell transfection. The day prior to transfection, cells were trypsinized, counted and seeded into 6-well plates at a density of $2 \times 10^{5}$ cells/well. When the cells reached $80 \%$ confluence, small interfering RNA (siRNA), miR-8112 mimic or inhibitor, AKT3 expression vector or negative control (NC) were transfected into cells using Lipofectamine ${ }^{\circledR} 2000$ (Invitrogen; Thermo Fisher Scientific, Inc.), according to the manufacturer's instructions. In a 6-well plate, cells at $80 \%$ confluence were transfected with $50 \mathrm{nM}$ miRs/siRNAs (miR-1182 mimic, inhibitor or NCs, siRNA and si-NC) or $6 \mu \mathrm{g}$ plasmids (AKT3 expression vector, sh-LINC01207 expression vector or empty vector control). After 16 -h incubation at $37^{\circ} \mathrm{C}$ with $5 \% \mathrm{CO}_{2}$, the transfection reagents were removed and replaced with fresh medium. Transfected cells were incubated for $48 \mathrm{~h}$ post-transfection in the cell culture incubator at $37^{\circ} \mathrm{C}$ with $5 \%$ $\mathrm{CO}_{2}$ before being used in subsequent experiments.

Plasmids AKT3 overexpression or shRNA transfection. The cDNA sequence of AKT3 was cloned into the pcDNA 3.1 plasmid (Suzhou GenePharma Co., Ltd.). The empty pcDNA 3.1 plasmid was used as NC. For sh-LINC01207 transfection, pLKO.1-Puro vector containing the sh-LINC01207 sequence or sh-NC were constructed by Guangzhou RiboBio Co., Ltd.

siRNA, miR-1182 mimic, miR-1182 inhibitor transfection. siRNA (si-NC, si-LINC01207\#1 and si-LINC01207\#2),
miR-1182 mimic, miR-1182 inhibitor and mimic-NC were obtained from Guangzhou RiboBio Co., Ltd. The following sequences were used: i) si-LINC01207\#1 sense, 5'-CCAGCT AAGACATTAGTAA-3' and antisense, 5'-TTACTAATGTCT TAGCTGG-3'; ii) si-LINC01207\#2 sense, 5'-GCAGGAAGG AATCCACAAT-3' and antisense, 5'-ATTGTGGATTCCTTC CTGC-3' (Guangzhou RiboBio Co., Ltd.); iii) si-NC sense, 5'-UGCUGACUCCAAAGCUCUGdTdT-3' and antisense, 5'-CAGAGCUUUGGAGUCAGCAdTdT-3' (Invitrogen; Thermo Fisher Scientific, Inc.); iv) miR-1182 mimic, 5'-GAG GGUCUUGGGAGGGAUGUGAC-3'; v) mimic-NC, 5'-CGG UACGAUCGCGGCGGGAUAUC-3'; vi) miR-1182 inhibitor sense, 5'-GAGGGUCUUGGGAGGGAUGUGAC-3' and antisense, 5'-GUCACAUCCCUCCCA AGACCCUC-3'; inhibitor-NC sense, 5'-GGUUCGUACGUACACUGUUCA-3' and antisense, 5'-UGA ACAGUGUACGUACGA ACC-3' (Sigma-Aldrich; Merck KGaA).

Reverse transcription-quantitative PCR (RT-qPCR). TRIzol ${ }^{\circledR}$ reagent (Invitrogen; Thermo Fisher Scientific, Inc.) was used to isolate total RNA according to the manufacturer's instructions. For cellular fractionation, Subcellular Fractionation Kit for Cultured Cells (Thermo Fisher Scientific, Inc.; cat. no. 78840) was used according to the manufacturer's instructions. Total RNA (1 $\mu \mathrm{g})$ was used to perform reverse transcription using Primer Script RT Master Mix kit (Takara Bio, Inc.; cat. no. RR036A) under following conditions: $30 \mathrm{~min}$ at $37^{\circ} \mathrm{C}, 5 \mathrm{~min}$ at $85^{\circ} \mathrm{C}$ and holding at $4^{\circ} \mathrm{C}$. qPCR analysis was performed using SYBR Select Master Mix (Applied Biosystems, Inc.; cat. no. 4472908) on the ABI 7900 system (Applied Biosystems, Inc.). The thermocycling conditions for the qPCR consisted of $30 \mathrm{sec}$ at $95^{\circ} \mathrm{C}$, followed by 40 cycles of $5 \mathrm{sec}$ at $95^{\circ} \mathrm{C}$ and $60 \mathrm{sec}$ at $60^{\circ} \mathrm{C}$. The relative gene expression levels were normalized against GAPDH gene using the $2^{-\triangle \Delta C q}$ method (30). The following PCR primers were used in the study: i) GAPDH forward, 5'-CCACATCGCTCAGAC ACCAT-3' and reverse, 5'-ACCAGGCGCCCAATACG-3'; ii) LINC01207 forward, 5'-CAGACACAGGCCATTCAG TC-3' and reverse, 5'-CTTCTTCACCAGAAGCATTCC-3'; iii) miR-1182 forward, 5'-GGGGAGGGTCTTGGGAGG GA-3' and reverse, 5'-GTGCAGGGTCCGAGGT-3'; iv) AKT3 forward, 5'-AATGGACAGAAGCTATCCAGGC-3' and reverse, 5'-TGATGGGTTGTAGAGGCATCC-3'; U6 forward, 5'-CTCGCTTCGGGCAGCACA-3' and reverse, 5'-AACGCC TTCCACGAATTTGCGT-3'.

Dual luciferase reporter assay. The bioinformatics website lncBase V.2 (https://rnacentral.org/expert-database/lncbase) was used to identify LINC01207 targets. The TargetScan database 7.2 (http://www.targetscan.org/vert_72/) was used to predict the target of miR-1182. The binding relationships between the identified targets were confirmed using dual luciferase reporter assays. The sequences of miR-1182 binding sites at the $3^{\prime}$ untranslated regions (UTRs) of LINC01207 and AKT3 were amplified and individually inserted into the pmirGlO luciferase reporter vector (Promega Corporation).

Wild-type (WT) and mutated (MUT) fragments containing the binding site were synthesized and cloned into a pMIR-REPORT plasmid (Guangzhou RiboBio Co., Ltd.). 
The plasmids were then co-transfected into PC-3 cells with miR-1182 mimic or mimic NC together with Renilla luciferase (hRlucneo) control plasmid (Promega Corporation). Before transfection, cells were seeded into a 96-well plate at a density of $2 \times 10^{3}$ cells/well. The target plasmid $(0.5 \mu \mathrm{g})$ and miR-1182 mimic $(0.5 \mu \mathrm{g})$ were mixed in $50 \mu \mathrm{l}$ serum-free Opti-MEM (Gibco; Thermo Fisher Scientific, Inc.). In a separate preparation, $1 \mu \mathrm{l}$ Lipofectamine 2000 was mixed in $50 \mu 1$ serum-free Opti-MEM medium. The two mixtures were combined together and incubated for $20 \mathrm{~min}$, before adding to the cells. A total of $48 \mathrm{~h}$ post-transfection, the relative luciferase activities were measured using Dual-Luciferase Reporter Assay kit (Promega Corporation; cat. no. E1910) on a luminescence microplate reader (Infinite 200 PRO; Tecan). The relative firefly luciferase activity in the reporter plasmid was normalized to that of Renilla luciferase in the hRlucneo control plasmid.

Cell Counting Kit-8 (CCK-8) proliferation assay. A CCK-8 assay was performed to detect changes in cell proliferation. PC-3 cells or PC-3-siLINC01207 cells were seeded into a 96-well plate at a density of $1 \times 10^{4}$ cells/well, and incubated at $37^{\circ} \mathrm{C}$ in $5 \% \mathrm{CO}_{2}$ for $48 \mathrm{~h}$. The CCK-8 (10 $\left.\mu \mathrm{l}\right)$ reagent was added to each well at $0,24,48$ and $72 \mathrm{~h}$ and incubated at $37^{\circ} \mathrm{C}$ for $1 \mathrm{~h}$. The GX71 microplate reader (Olympus Corporation) was used to detect the absorbance value (optical density value) in each well at $450 \mathrm{~nm}$.

Colony formation assay. PC-3 cells or PC-3-si-LINC01207 cells were seeded into a 6-well plate at a density of 500 cells/well after cell counting. The culture medium was changed every 2 days. After 14 days of culture, the cells were fixed in $100 \%$ methanol for $30 \mathrm{~min}$ at room temperature and stained for 10 min using Giemsa Stain kit (Abcam; cat. no. ab150670) according the manufacturer's instructions. After 14 days, an image of the cell colony was captured with a digital camera using an AM6000 microscope (Leica Microsystems, Inc.). Colonies consisting of $>50$ cells were counted manually.

Apoptosis detection using flow cytometry. The Annexin-V-FITC apoptosis assay kit (BD Biosciences) was utilized to analyze cell apoptosis. PC 3 cells $\left(2 \times 10^{5}\right)$ transfected with si-LINC01207\#1, si-LINC01207\#2 or si-NC were harvested at $48 \mathrm{~h}$ after transfection. Cells were collected after trypsinization and resuspended in Annexin $\mathrm{V}$ binding buffer. The solution containing $1 \mathrm{X}$ Annexin-V-FITC/propidium iodide (PI) staining reagent was used to stain cells for $15 \mathrm{~min}$ at $37^{\circ} \mathrm{C}$. The FACSCalibur flow cytometer (BD Biosciences) was utilized to detect cell apoptosis. The data were analyzed using FlowJo software 7.6 (FlowJo LLC).

Western blot analysis. Total protein lysates were harvested in cold NP40 lysis buffer (1 mM EDTA, $150 \mathrm{mM} \mathrm{NaCl}, 1 \%$ NP-40, $20 \mathrm{mM}$ Tris-HCl, phosphatase/protease inhibitor cocktail, pH 7.4). A Bradford assay was used to measure protein concentration. Total protein $(30 \mu \mathrm{g})$ was separated by $4-12 \%$ gradient SDS-PAGE and transferred onto a polyvinylidene fluoride membranes. The membrane was blocked with $5 \%$ skimmed milk for $2 \mathrm{~h}$ at room temperature, then incubated overnight with primary antibodies (dilution, $1: 1,000$ in PBS) at $4^{\circ} \mathrm{C}$. The membranes were then washed with TBS with 5\% Tween-20 three times, then incubated with horseradish peroxidase (HRP)-conjugated goat anti-mouse IgG secondary antibody at room temperature for $90 \mathrm{~min}$. The antibodies used in the study were anti-AKT3 (dilution, 1:1,000; cat. no. ab152157), anti-GAPDH (6C5 Loading Control; dilution, 1:1,000; cat. no. ab8245) and rabbit anti-mouse IgG H\&L (dilution, 1:2,500; cat. no. ab6728) (all from Abcam). Protein bands were developed using Pierce $^{\mathrm{TM}}$ ECL Plus-Chemiluminescent HRP Substrates kit (Thermo Fisher Scientific, Inc.) followed by visualization using the iBright ${ }^{\mathrm{TM}}$ FL1500 Imaging System (Thermo Fisher Scientific, Inc.). Relative protein expression was normalized to that of GAPDH using ImageJ software V 1.8.0 (National Institutes of Health).

Lentivirus-mediated shRNA stable expression cell line generation. The recombinant lentivirus vectors expressing shRNA targeting LINC01207 and the $0.45-\mu \mathrm{m}$ nitrocellulose (NC) membrane filter were prepared by Shanghai GeneChem Co., Ltd. A total of $12 \mu \mathrm{g}$ third generation packaging vectors pVSV-G and pGag-Pol (Addgene, Inc.), and $10 \mu \mathrm{g}$ shRNA expression vector pLenti-puro-shLINC0120 (Addgene, Inc.) were transfected into 293T cells (American Type Culture Collection) in a $10-\mathrm{cm}$ cell culture dish with Lipofectamine 2000 according to the manufacturer's instruction. The same amount of empty vector pLenti-puro (Addgene, Inc.) was used to produce negative control lentivirus (sh-NC group). Transfected cells were incubated for $48 \mathrm{~h}$ at $37^{\circ} \mathrm{C}$. The cell culture supernatant containing the lentivirus was collected $48 \mathrm{~h}$ after transfection and was filtered with a $0.45-\mu \mathrm{m}$ nitrocellulose membrane filter. The lentiviral supernatant was stored at $-80^{\circ} \mathrm{C}$ until further use. To generate stable cell lines, PC3 cells were infected with the recombinant lentivirus at a MOI of 5 in the presence of $10 \mu \mathrm{g}$ polybrene (Sigma-Aldrich; Merck KGaA; cat. no. tr-1003-g). Infected cells were selected with $1.0 \mu \mathrm{g} / \mathrm{ml}$ puromycin for two weeks to eliminate the uninfected cells before further experiment. qPCR was performed to confirm the efficient knockdown of LINC01207.

In vivo xenograft model. BALB/c immunodeficient nude mice (4-6 weeks old; 18-25 g) were provided by Experimental Animal Center, College of Veterinary Medicine, Yangzhou University (Yangzhou, China). Mice was raised under the pathogen-free condition. The Institutional Committee for Animal Care and Use approved the present study protocols. All the animal procedures were conducted strictly following the approved protocols. PC-3-sh-NC and PC-3-sh-LINC01207 cells $\left(2 \times 10^{6}\right)$ were re-suspended in Matrigel ${ }^{\mathrm{TM}}$, and injected subcutaneously into the flanks of BALB/c mice. Tumor volume and mouse body weight were recorded on 7, 14, 21, 28, 35, 42 day. On day 42, all mice were euthanized and tumors were collected, weighed and imaged. A total of 6 mice were included in sh-NC and sh-LINC01207 group. Carbon dioxide was used for euthanasia. Briefly, a euthanizing chamber was connected to a carbon dioxide cylinder and the flow rate was adjusted to displace $20 \%$ of the cage volume per min. Mice were placed into the euthanizing chamber for $10 \mathrm{~min}$ 
until no movement was observed. Death was assured by subsequent by cervical dislocation. The tumors of terminally dead mice were resected for weight measurement. All animal experiments followed the Guide for the Care and Use of Laboratory Animals (US and National Institutes of Health) (31). All animal procedures were approved by the Animal Care and Use Ethical Committee of Central Hospital of Enshi Tujia and Miao Autonomous Prefecture, Wuhan, China (approval no. 20176209).

The Cancer Genome Atlas (TCGA) datasets. TCGA data consisting of $492 \mathrm{PC}$ tumor tissues and 152 normal prostate tissues was downloaded from GEPIA database (http://gepia. cancer-pku.cn/). Gene expression profiles from TCGA were utilized to compare LINC01207 gene expression between PC tumor and normal prostate tissue.

Patient tissue collection. A total of 50 PC tumor samples and 50 matched non-carcinoma tissue samples $(\sim 2 \mathrm{~cm}$ away from the primary tumor site) were obtained from 50 male patients by surgery from January 2018 to December 2019 in Enshi Central Hospital (Wuhan, China). All patients were initially diagnosed by biopsy examination by experienced pathologists. The age of the 50 enrolled patients ranged between 35 and 61 years, with a median age of 48 years. Patients meeting the following criteria were enrolled: i) Patients confirmed by tumor pathology and genetics; ii) patients without other histories of urologic neoplasms or tumor; and iii) patients who received no chemotherapy or radiotherapy before the operation. Patients meeting the following criteria were excluded: i) Patients with severe impairment of vital organs, such as heart, liver and lung; ii) patients with autoimmune history; iii) patients with autoimmune history; and iv) patients with chronic infectious disease or acute contagious diseases. The tissues were harvested and kept in liquid nitrogen before further experiment. All patients involved in the study were informed of the details and completed written informed consent forms. The patients did not experience radiotherapy and chemotherapy before surgery. This study was approved by the Medical Ethics Committee of Central Hospital of Enshi Tujia and Miao Autonomous Prefecture, Wuhan, China (approval no. 20176209).

Statistical analysis. GraphPad Prism 5 (GraphPad Software, Inc.) was used for statistical analysis. All experiments were repeated three times, and the results are presented as the mean \pm SEM. Differences between PC and adjacent normal tissue samples were analyzed using paired two-tailed Student's t-tests. In all other cases where two groups were compared, two unpaired groups were compared using unpaired t-tests. Comparisons of continuous variables among multiple groups were analyzed using one-way ANOVA followed by Tukey's post hoc test for pairwise comparisons. Comparisons of data with effect of two nominal predictor variables were performed using two-way ANOVA. Kaplan-Meier and log-rank tests were used to compare the cumulative survival rates. Pearson's correlation analysis was used to analyze correlation between gene expression levels $\mathrm{P}<0.05$ was considered to indicate a statistically significant difference.

\section{Results}

Expression of LINC01207 is upregulated and correlated with poor prognosis in PC patients. To determine LINC01207 expression level in PC tumors, TCGA data consisting of 492 PC patients and 152 normal prostate tissues were downloaded. Expression analysis demonstrated that LINC01207 expression was significantly increased in PC tumor samples compared with non-carcinoma tissue samples (Fig. 1A). To confirm this result, $50 \mathrm{PC}$ tumor samples and 50 matched normal samples were collected, and LINC01207 expression using RT-qPCR. LINC01207 showed a significantly higher expression level in PC samples relative to the matched normal samples (Fig. 1B). LINC01207 expression levels were also examined in human PC cell lines (LNCaP, PC-3, C4-2B and Du-145) and a normal prostate cell line (RWPE1). RT-qPCR analysis showed that LINC01207 was upregulated in all PC cell lines compared with the RWPE1 cell line. Among the PC cell lines, LINC01207 showed highest expression in PC-3 cells (Fig. 1C). Furthermore, the median expression value of LINC01207 in PC tissues was used as the cut-off to divide the 50 patients with PC into high- $(n=25)$ and low-expression $(n=25)$ groups. Kaplan-Meier survival curves were used to evaluate overall survival rate of two groups of patients. Our analysis showed that high levels of LINC01207 was associated with prognosis (Fig. 1D). Collectively, these data showed that LINC01207 expression was significantly increased in PC tumor tissue, suggesting an oncogenic role of LINC01207 in PC.

Knockdown of LINC01207 inhibits the aggressive phenotype of PC cell. LINC01207 expression showed highest upregulation in PC-3 cell line as indicated in Fig. 1C; therefore, the PC-3 cell line was chosen for further study. LINC01207 was knocked down in PC-3 cells using siRNA to assess its biological function. RT-qPCR analysis demonstrated that si-LINC01207\#1 and si-LINC01207\#2 could efficiently decrease LINC01207 expression in PC-3 cells (Fig. 2A). CCK-8 assays were then used to determine the role of LINC01207 in PC-3 proliferation. Both LINC01207\#1 and si-LINC01207\#2 significantly suppressed the growth of PC-3 cells (Fig. 2B). Consistently, LINC01207\#1 and si-LINC01207\#2 impaired the colony formation ability in PC-3 cells (Fig. 2C). Furthermore, Annexin V/PI staining showed that silencing LINC01207 significantly increases the percentage of apoptotic events in PC-3 cells. To further evaluate the role LINC01207 in tumorigenesis, stable cell lines expressing shRNA were generated (PC-3-shNC and PC-3-shLINC01207 cells), which were inoculated subcutaneously in nude mice and the tumor volume was continuously monitored. The silencing efficiency of shLINC01207 was validated by RT-qPCR (Fig. S1A). Silencing of LINC01207 significantly suppressed the tumor growth rate (Fig. 2E). Meanwhile, tumor weight was significantly smaller in LINC01207 silenced group (Fig. 2F).

Taken together, these results indicate that LINC01207 is indispensable for proliferation, colony formation and cell survival of PC-3 cells in vitro. It is also required to support the tumorigenesis in the xenograft mouse model.

LINC01207 sponges miR-1182. To explore the molecular mechanisms of LINC01207 in regulating the aggressive 
A

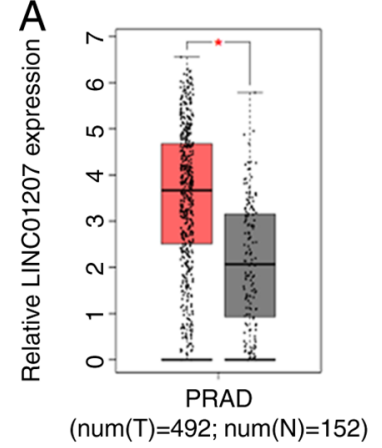

$\mathrm{B}$

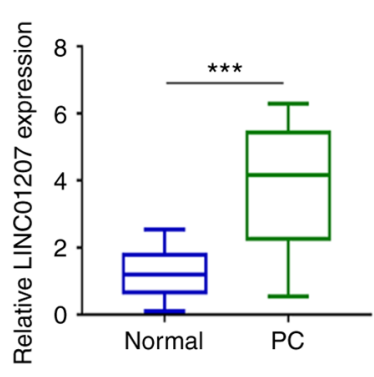

C

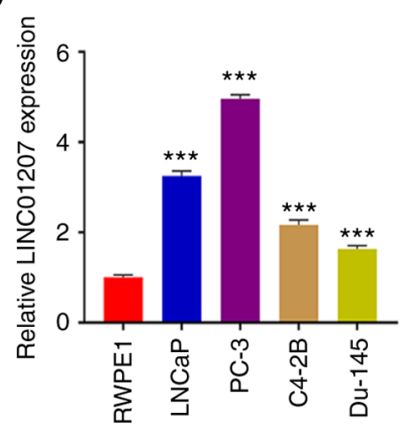

$\mathrm{D}$

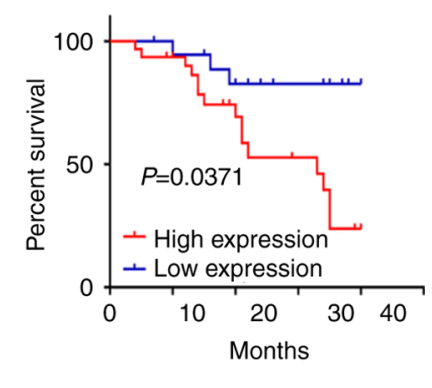

Figure 1. LINC01207 expression is upregulated and associated with poor prognosis in patients with PC. (A) LINC01207 expression in PC tumor tissues ( $\mathrm{n}=492$ ) and normal tissues ( $\mathrm{n}=152$; non-matched from a different cohort) from The Cancer Genome Atlas. (B) LINC01207 levels in PC tumor tissue and adjacent normal tissues. $\mathrm{n}=50$ in each group. (C) LINC01207 levels in PC cell lines and a normal prostate cell line. (D) The median expression value of LINC01207 in $\mathrm{PC}$ tissues was used as the cut-off to divide the 50 patients with PC into high- $(\mathrm{n}=25)$ and low-expression $(\mathrm{n}=25)$ groups. ${ }^{*} \mathrm{P}<0.05,{ }^{* * * *} \mathrm{P}<0.001 . \mathrm{LINC} 01207$, long intergenic non-protein coding RNA 1207; PC, prostate cancer.

A

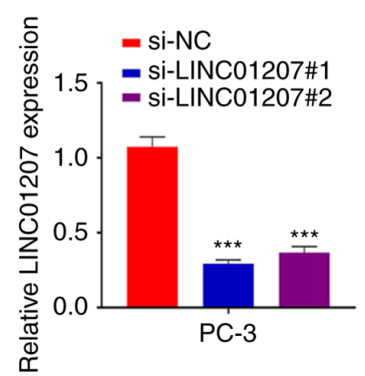

$\mathrm{D}$

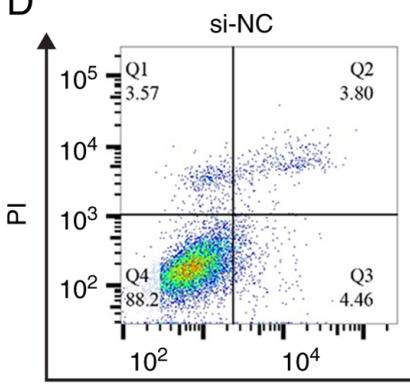

B

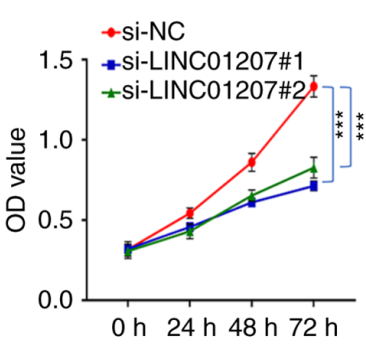

Si-LINC01207\#1

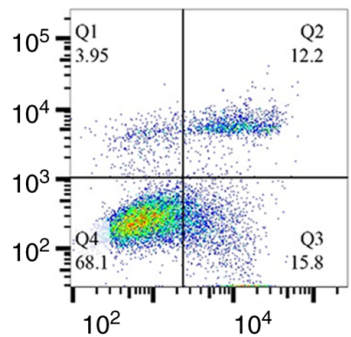

C
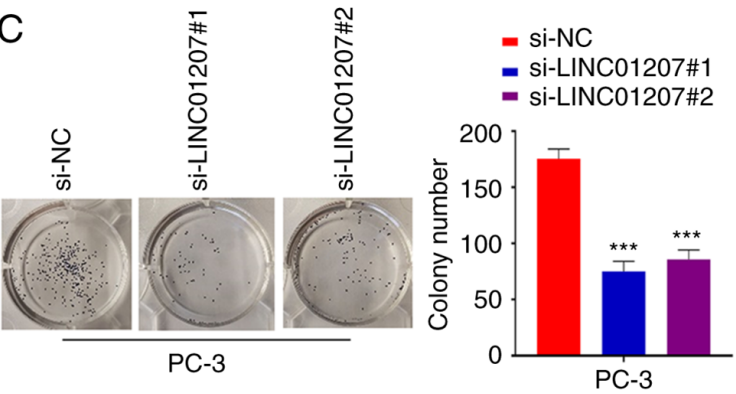
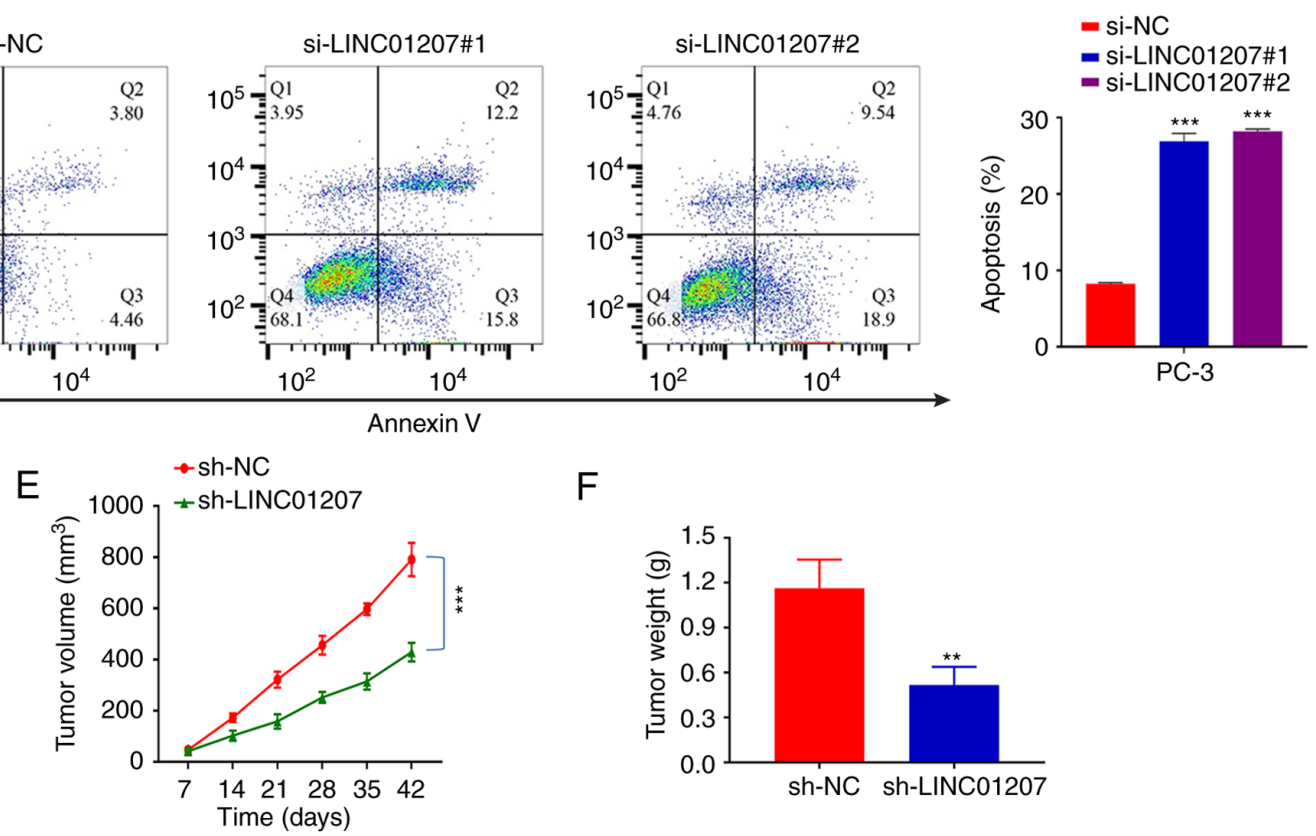

$\mathrm{F}$

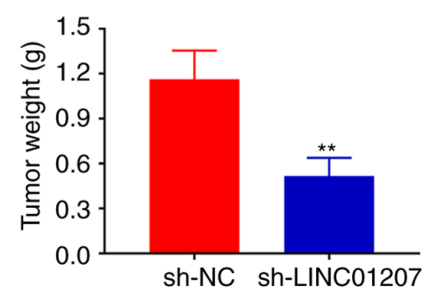

Figure 2. Knockdown of LINC01207 inhibits aggressive phenotype in prostate cancer cells. (A) LINC01207 was knocked down in PC-3 cells using si-LINC01207\#1 and si-LINC01207\#2. (B) Effects of LINC01207 knockdown on PC-3 cell proliferation. (C) Effects of LINC01207 knockdown on PC-3 cell colony forming ability. (D) Effects of LINC01207 knockdown on the apoptosis of PC-3 cells. (E) PC-3 cells were infected with lentivirus carrying shRNA targeting LINC01207 or sh-NC. The cells were injected into nude mice, and the tumor volume was monitored. (F) Knockdown of LINC01207 negatively affected tumor weight. ${ }^{* *} \mathrm{P}<0.01,{ }^{* * *} \mathrm{P}<0.001$. LINC01207, long intergenic non-protein coding RNA 1207; small interfering RNA; shRNA, short hairpin RNA; $\mathrm{NC}$, negative control; OD, optical density; PI, propidium iodide.

behavior of PC cells, its target miRNA was examined. First, nuclear and cytoplasmic fractions were separated in PC-3 cells to detect the cellular sub-localization of LINC01207.
As revealed by RT-qPCR analysis, LINC01207 was mostly enriched in the cytoplasm (Fig. 3A). Next, the miRNA targets of LINC01207 were predicted using the IncBase online tool, 

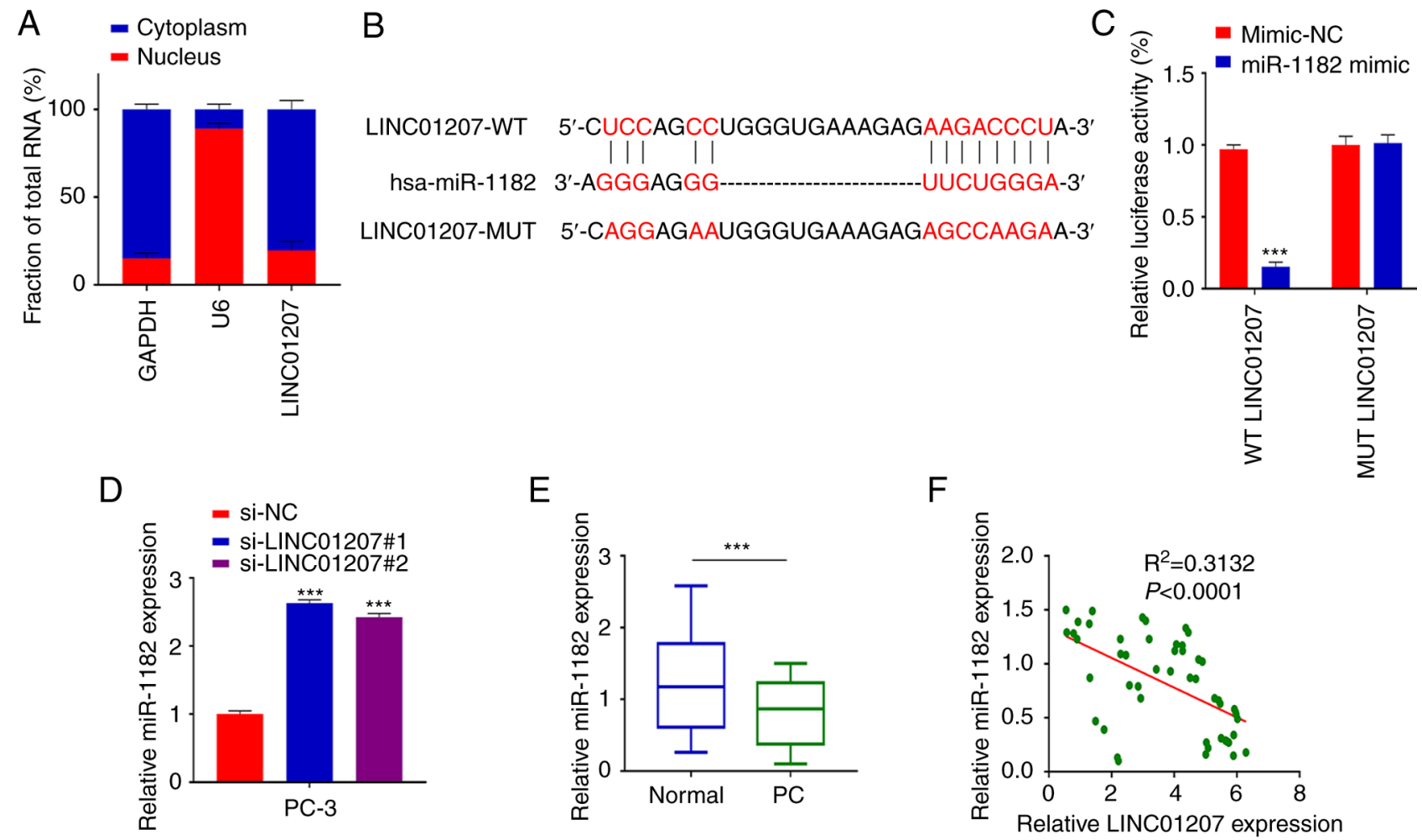

Figure 3. LINC01207 sponges miR-1182. (A) LINC01207 is mainly located in the cytoplasm. Nuclear and cytoplasmic fractions were extracted from PC-3, and LINC01207 expression was measured. GAPDH and U6 were used as cytoplasmic and nuclear controls. (B) Schematic representation of the LINC01207 target site in miR-1182 mRNA. (C) Transfection with the miR-1182 mimic inhibited the luciferase activity of WT LINC01207, but not MUT LINC01207. (D) The expression of miR-1182 was upregulated after LINC01207 knockdown in PC-3 cells. (E) miR-1182 expression levels in PC tumor and adjacent normal tissue samples. $\mathrm{n}=50$ in each group. (F) Pearson's correlation analysis was used to analyze the expression levels of miR-1182 and LINC01207. ${ }^{* * *} \mathrm{P}<0.001$. LINC01207, long intergenic non-protein coding RNA 1207; PC, prostate cancer; miR, microRNA; hsa, Homo sapiens; WT, wild-type; MUT, mutant; NC, negative control.

which identified miR-1182 as a potential target binding to LINC01207 (Fig. 3B). To confirm their functional interaction, a dual luciferase reporter assay was performed in the presence of miR-1182 mimic or mimic-NC. Transfection of miR-1182 mimic significantly increased intracellular miR-1182 level in PC-3 cells (Fig. S1B). miR-1182 mimic significantly suppressed the luciferase reporter activity when compared with mimic-NC, which was abrogated in the luciferase reporter with mutated binding site (Fig. 3C). Moreover, silencing LINC01207 could downregulate miR-1182 expression in PC-3 cells (Fig. 3D). In PC tumor samples and matched normal samples, miR-1182 expression showed significantly lower level in PC tumor tissues (Fig. 3E). Furthermore, Pearson's correlation analysis revealed a negative correlation between the expression levels of LINC01207 and miR-1182 (Fig. 3F). Taken together, these data indicate that LINC01207 interacts with and negatively regulates miR-1182.

AKT3 is downstream target gene of miR-1182. The gene target of miR-1182 was then identified. TargetScan was used to predict the miR-1182 target genes. As a result, AKT3 was identified as a potential miR-1182 target gene (Fig. 4A). To confirm their functional interaction, a dual luciferase reporter assay was performed in the presence of miR-1182 mimic or mimic-NC. miR-1182 mimic significantly inhibited luciferase reporter of wild-type AKT3 3'UTR but not the reporter containing mutated binding site (Fig. 4B). miR-1182 mimic also decreased AKT3 expression at both mRNA and protein level (Fig. 4C).
Consistently, knockdown of miR-1182 by inhibitor significantly lowered the level of miR-1182 (Fig. S1C), and increased AKT3 expression at both mRNA and protein level (Fig. 4C). AKT3 was also upregulated in PC tumor tissue compared with matched normal tissue samples (Fig. 4D). Of note, the expression levels of AKT3 and miR-1182 were negatively correlated. By contrast, the expression levels of AKT3 and LINC01207 were positively correlated in 50 pairs of PC tumor tissues and matched normal tissues. Altogether, these data suggest that ATK3 is a target of miR-1182, and the expression levels of these two molecules are negatively correlated in PC.

LINC01207 sponges miR-1182 to sustain AKT3 level in PC cells. The above data showed that LINC01207, miR-1182 and AKT3 expression are inter-correlated with each other in PC tumors. Transfection with si-NC, si-LINC01207\#1, miR-1182 inhibitor, si-LINC01207\#1+miR-1182 inhibitor and si-LINC01207\#1 +AKT3 was used in PC3 cells to validate a functional role of LINC01207/ miR-1182/AKT3 axis. qPCR analysis showed that transfection of AKT3 expression vector in PC-3 cells increased intracellular AKT3 mRNA level by about 2.5 times (Fig. S1D). As revealed by WB analysis, LINC01207 silencing decreased AKT3 protein expression; conversely, AKT3 level was elevated in the presence of miR-1182 inhibitor. The presence of miR-1182 inhibitor or AKT3 expression vector partially rescued AKT3 level downregulated by si-LINC01207\#1 (Fig. 5A). LINC01207 and miR-1182 expression levels were also examined. LINC01207 
A

$\begin{array}{lr}\text { AKT3 3'UTR-WT } & \text { 5'-GAUGAAGGUCUCAGACCCUG-3' } \\ \text { hsa-miR-1182 } & \text { 3'-CAGUGUAGGGAGGGUUCUGGGAG-5' } \\ \text { AKT3 3'UTR-MUT } & \text { 5'-GAUGAAGGUCUCGACCAGGG-3' }\end{array}$

$\mathrm{B}$

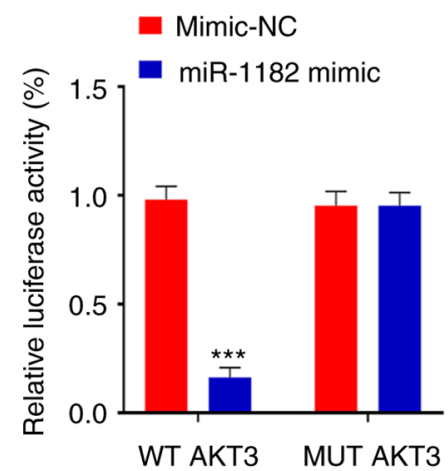

C

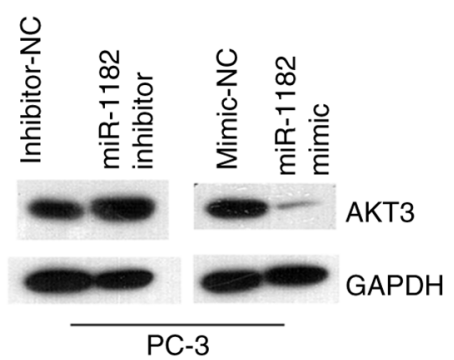

D

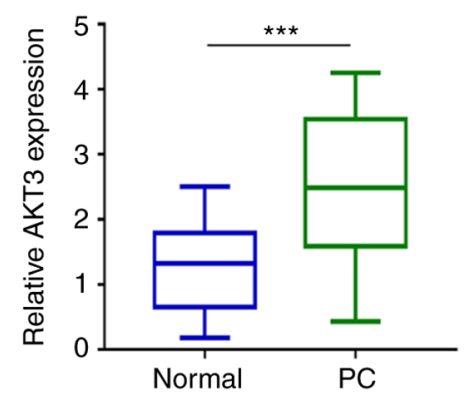

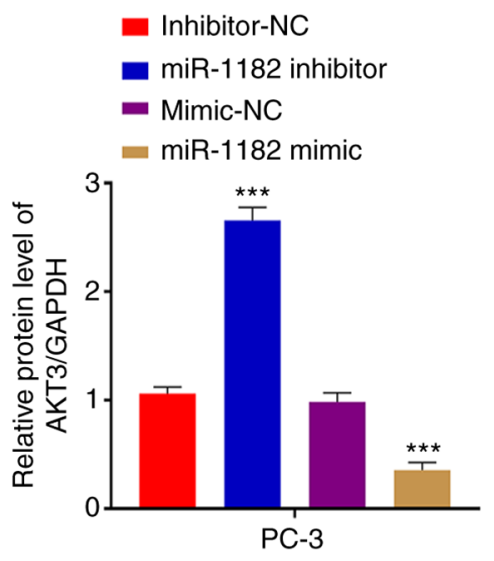

E

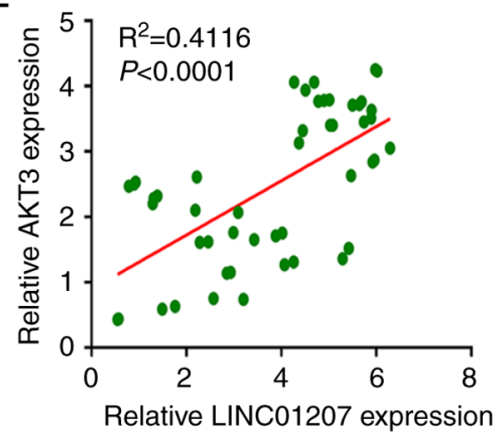

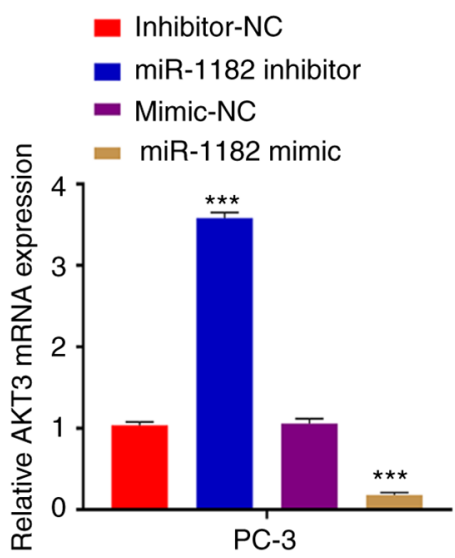

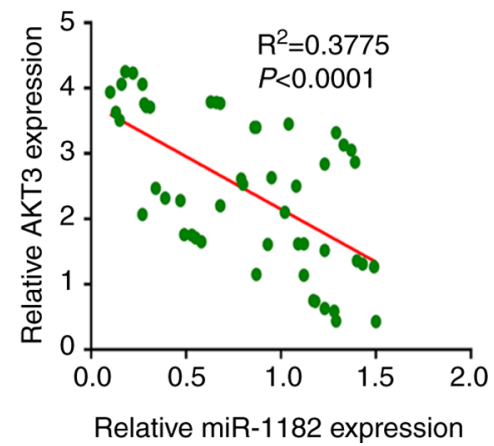

Figure 4. AKT3 was validated as a target gene of miR-1182. (A) AKT3 was identifies as the target gene of miR-1182 using the TargetScan database. (B) Transfection with the miR-1182 mimic inhibited luciferase activity of WT AKT3 reporter, but not MUT AKT3. (C) AKT3 mRNA and protein expression was downregulated following transfection with miR-1182 mimic and upregulated following transfection with miR-1182 inhibitor. (D) AKT3 mRNA expression levels in PC and adjacent normal tissue sample. $n=50$ in each group (E) AKT3 expression negatively correlates with that of miR-1182 and positively correlates with that of LINC01207 PC and adjacent normal tissue samples. $\mathrm{n}=50$ in each group. ${ }^{* * *} \mathrm{P}<0.001$. LINC01207, long intergenic non-protein coding RNA 1207; PC, prostate cancer; miR, microRNA; hsa, Homo sapiens; WT, wild-type; MUT, mutant; NC, negative control.

silencing increased miR-1182 mRNA expression, while LINC01207 expression was dramatically increased when cell was treated with miR-1182 inhibitor (Fig. 5B). Moreover, both LINC01207 and miR-1182 levels were slightly increased when cells were co-transfected si-LINC01207\#1 with miR-1182 inhibitor or AKT3 (Fig. 5B). CCK8 proliferation assay showed that the presence of miR-1182 inhibitor or AKT3 expression vector partially rescued the cellular proliferation which was suppressed by si-LINC01207\#1 (Fig. 5C). Consistently, the presence of miR-1182 inhibitor or AKT3 expression vector also rescued the colony formation ability when suppressed LINC01207 was silenced (Fig. 5D). Finally, apoptosis assay detected by FACS revealed that the presence of miR-1182 inhibitor or AKT3 expression vector significantly recued the apoptotic events induced by LINC01207 silencing (Fig. 5E). Collectively, these data imply that LINC01207 plays an oncogenic role in PC cells by negatively regulating miR-1182 to maintain a high level of AKT3.

\section{Discussion}

$\mathrm{PC}$ is the most prevalent malignancy in male patient globally and the 4th leading mortality in cancer (32). Accumulating evidence has shown that lncRNA is involved in tumorigenesis and cancer progression $(33,34)$. Abnormal miRNA expression is another factor contributing to tumorigenesis by dysregulating target genes related to PC progression $(14,15)$. Meanwhile, several studies have revealed that lncRNA 
A

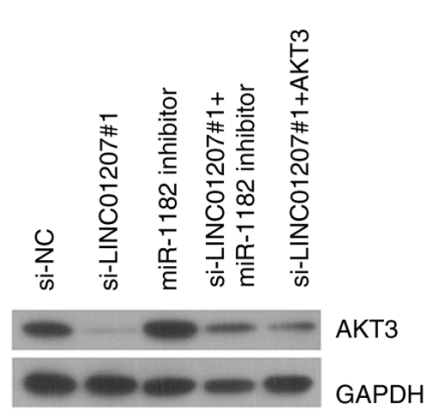

B
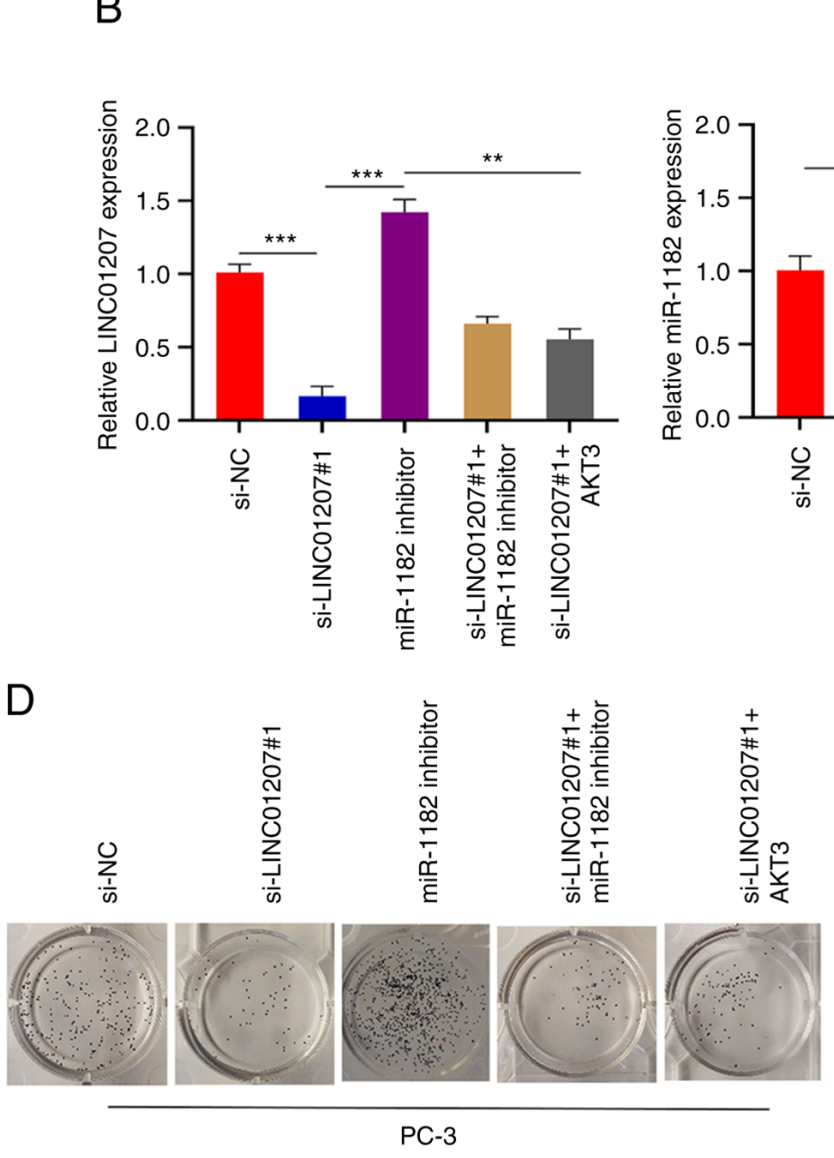
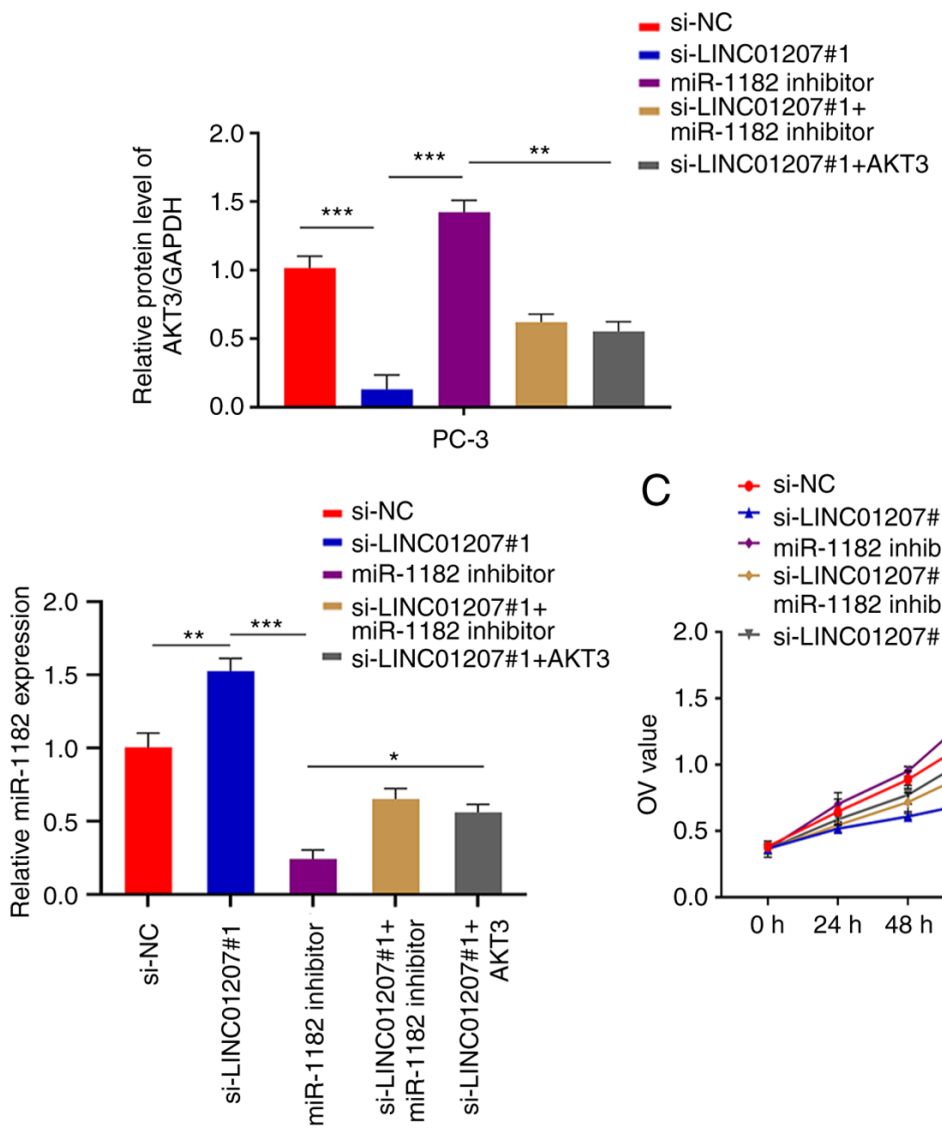

$\mathrm{C} \rightarrow \mathrm{si}-\mathrm{NC}$

- si-LINC01207\#1

$\rightarrow$ miR-1182 inhibitor

$\rightarrow$ si-LINC01207\#1+

miR-1182 inhibitor

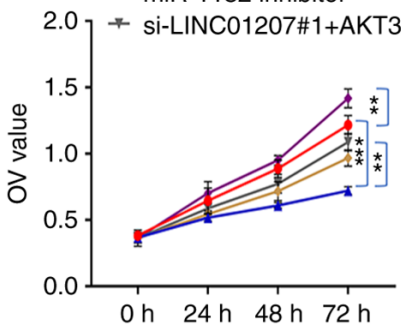

E $=$ si-NC

- si-NC

- si-LINC01207\#1

- miR-1182 inhibitor

- si-LINC01207\#1+ miR-1182 inhibitor

- si-LINC01207\#1+AKT3
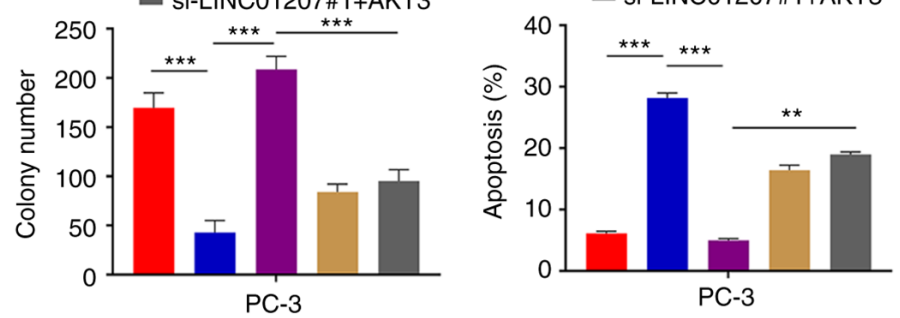

si-LINC01207\#1+ AKT3

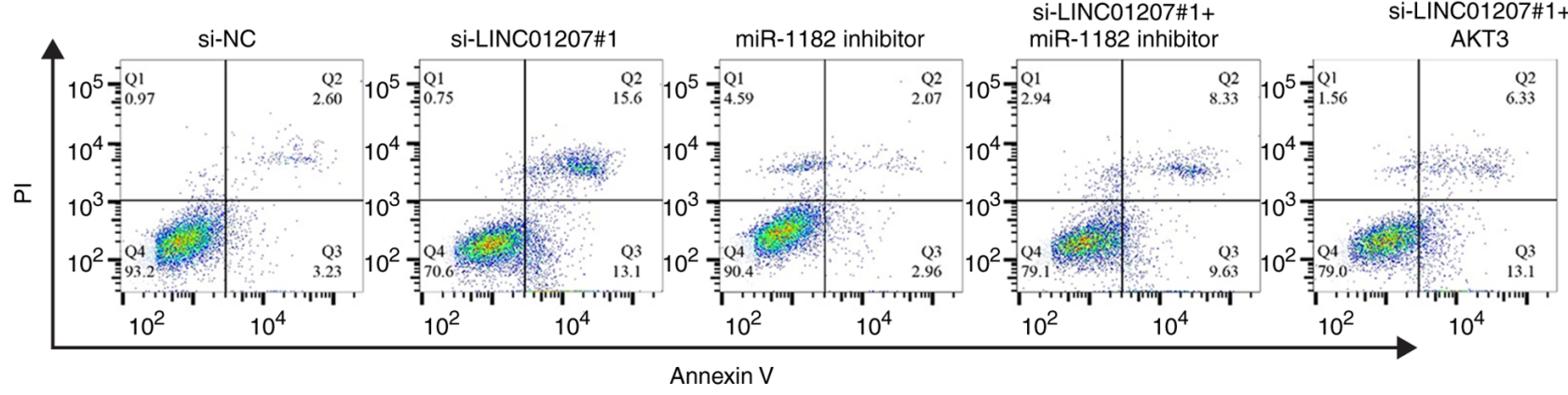

Figure 5. LINC01207 sponges miR-1182 to maintain AKT3 in prostate cancer cells. PC-3 cells were transfected with si-NC, si-LINC01207\#1, miR-1182 inhibitor, si-LINC01207\#1 + miR-1182 inhibitor or si-LINC01207\#1 + AKT3. (A) AKT3 protein expression levels following transfection. (B) LINC01207 and miR-1182 expression following transfection. (C) Proliferation following transfection. (D) Colony-forming following transfection. (E) Apoptosis rate in PC-3 cells following transfection. ${ }^{*} \mathrm{P}<0.05,{ }^{* * *} \mathrm{P}<0.01,{ }^{* * *} \mathrm{P}<0.001$. LINC01207, long intergenic non-protein coding RNA 1207; miR, microRNA; NC, negative control; si, small interfering RNA; OD, optical density; PI, propidium iodide.

promotes PC growth mainly by regulating genes and pathways supporting cell proliferation (1,3-5). Several miRNA molecules and other proteins such as kallikreins have been proposed as PC biomarkers (6). In addition, LINC01207 was 
previously reported to be highly expressed in prostate cancer, as it could downregulate miR-1972 and upregulate LIM and $\mathrm{SH} 3$ protein 1 to promote $\mathrm{PC}$ progression (35). In the present study, LINC01207 seems to act as an oncogenic gene through modulating miR-1182/AKT3 axis. Collectively, previous search and our results support the notion that LINC01207 plays a functional role for the progression of PC.

Aberrant expression of LINC01207 has been reported in numerous cancer types, such as pancreatic cancer and lung adenocarcinoma $(26,29)$. The present study adds novel evidence of the abnormal LINC01207 expression in patients with PC, which is associated with the poor prognosis. A previous study has suggested that several upregulated lncRNA molecules seem to predict a dismal prognostic outcome of PC (33). LINC01207 seems to be required to support the proliferation and survival of $\mathrm{PC}$ cells both in vitro and in vivo. Therefore, the present study and a previous report (33) suggest that a high level of LINC01207 expression could be used as a biomarker to predict poor prognosis in PC patients.

The present study identified miR-1182 as a target of LINC01207. miR-1182 dysregulation has been widely reported in a variety of cancers, such as bladder cancer (16), ovarian cancer (17), gastric cancer (24) and PC (19). Therefore, miR-1182 may plays a ubiquitous role in the regulating tumorigenesis of different types of cancer. However, indifferent type of cancer, the exact molecular mechanisms underlying its regulation can be different. In PC cells, the present study demonstrated that the upregulation of LINC01207 negatively impacted on miR-1182 expression. Impaired expression of miR-1182 was also observed in patients with PC. These results suggest that miR-1182 may function as a tumor-suppressor gene, which antagonizes the oncogenic effect of LINC01207.

Previous studies also showed high expression of AKT3 in PC tumors, which highlights AKTs as oncogenic factors in PC progression $(23,24)$. AKT signaling is an important metabolic modulator coordinating cell survival and growth, which is frequently dysregulated in tumor cells $(23,24)$. The present study showed miR-1182 targets and negatively regulates AKT3 in PC cells. Since ATK3 expression positively correlates with LINC01207 but negatively correlates with miR-8812, and AKT3 is negatively regulated by miR-8812, it may be proposed that LINC01207 sustains AKT3 expression by sponging miR-8812 to support PC cell proliferation and PC progression. Modulating the LINC01207/miR-1182/AKT3 axis could be a novel strategy to control PC progression, which needs to be further evaluated in mouse models.

Overall, the findings of the present study indicate that LINC01207 serves as an oncogenic factor that is upregulated PC tumors. The aberrant upregulation of LINC01207 negatively impacts on miR-1182 to sustain the expression of AKT3, which supports the tumorigenesis of PC cells. LINC01207 knockdown suppresses proliferation, induces apoptosis and impairs the tumorigenesis of PC cells. Targeting the LINC01207/miR-1182/AKT3 axis could be leveraged as a potential therapeutic strategy in the treatment of PC.

\section{Acknowledgements}

Not applicable.

\section{Funding}

No funding was received.

\section{Availability of data and materials}

The datasets used and/or analysed during the current study are available from the corresponding author on reasonable request.

\section{Authors' contributions}

DQ and CN designed and performed the experimental work. $\mathrm{BD}$ and $\mathrm{ZH}$ performed literature research, research design and manuscript editing. BT and $\mathrm{SH}$ performed the statistical analysis. DQ and CN confirm the authenticity of all the raw data. All authors read and approved the final manuscript.

\section{Ethics approval and consent to participate}

All animal procedures were approved by the Animal Care and Use Ethical Committee of Central Hospital of Enshi Tujia and Miao Autonomous Prefecture (approval no. 20176209; Wuhan, China). All patients enrolled in the study were informed of the details and provided written informed consent. The use of the clinical samples was approved by The Medical Ethics Committee of Central Hospital of Enshi Tujia and Miao Autonomous Prefecture (approval no. 20176209).

\section{Patient consent for publication}

Not applicable.

\section{Competing interests}

The authors declare that they have no competing interests.

\section{References}

1. Edwards DR, Moroz K, Zhang H, Mulholland D, Abdel-Mageed AB and Mondal D: PRL-3 increases the aggressive phenotype of prostate cancer cells in vitro and its expression correlates with high-grade prostate tumors in patients. Int J Oncol 52: 402-412, 2018.

2. Shukla ME, Yu C, Reddy CA, Stephans KL, Klein EA, Abdel-Wahab M, Ciezki J and Tendulkar RD: Evaluation of the current prostate cancer staging system based on cancer-specific mortality in the surveillance, epidemiology, and end results database. Clin Genitourin Cancer 13: 17-21, 2015.

3. Miyake $\mathrm{H}$ and Fujisawa $\mathrm{M}$ : Prognostic prediction following radical prostatectomy for prostate cancer using conventional as well as molecular biological approaches. Int J Urol 20: 301-311, 2013.

4. Heidenreich A, Bellmunt J, Bolla M, Joniau S, Mason M, Matveev V, Mottet N, Schmid HP, van der Kwast T, Wiegel T, et al: EAU guidelines on prostate cancer. Part 1: Screening, diagnosis, and treatment of clinically localised disease. Eur Urol 59: 61-71, 2011.

5. Dall'Era MA, Albertsen PC, Bangma C, Carroll PR, Carter HB, Cooperberg MR, Freedland SJ, Klotz LH, Parker C and Soloway MS: Active surveillance for prostate cancer: A systematic review of the literature. Eur Urol 62: 976-983, 2012

6. Egidi MG, Cochetti G, Guelfi G, Zampini D, Diverio S, Poli G and Mearini E: Stability assessment of candidate reference genes in urine sediment of prostate cancer patients for miRNA applications. Dis Markers 2015: 973597, 2015. 
7. Cochetti G, Poli G, Guelfi G, Boni A, Egidi MG and Mearini E: Different levels of serum microRNAs in prostate cancer and benign prostatic hyperplasia: Evaluation of potential diagnostic and prognostic role. Onco Targets Ther 9: 7545-7553, 2016.

8. Bartel DP: MicroRNAs: Genomics, biogenesis, mechanism, and function. Cell 116: 281-297, 2004.

9. Calin GA and Croce CM: MicroRNA signatures in human cancers. Nat Rev Cancer 6: 857-866, 2006.

10. Gao Q and Zheng J: microRNA-323 upregulation promotes prostate cancer growth and docetaxel resistance by repressing p73. Biomed Pharmacother 97: 528-534, 2018.

11. Kanwal R, Plaga AR, Liu X, Shukla GC and Gupta S: MicroRNAs in prostate cancer: Functional role as biomarkers. Cancer Lett 407: 9-20, 2017.

12. Cochetti G, Rossi de Vermandois JA, Maulà V, Giulietti M, Cecati M, Del Zingaro M, Cagnani R, Suvieri C, Paladini A and Mearini E: Role of miRNAs in prostate cancer: Do we really know everything? Urol Oncol 38: 623-635, 2020.

13. Guelfi G, Cochetti G, Stefanetti V, Zampini D, Diverio S, Boni A and Mearini E: Next generation Sequencing of urine exfoliated cells: An approach of prostate cancer microRNAs research. Sci Rep 8: 7111, 2018.

14. Situ J, Zhang H, Jin Z, Li K, Mao Y and Huang W: MicroRNA-939 directly targets HDGF to inhibit the aggressiveness of prostate cancer via deactivation of the WNT/ $\beta$-catenin pathway. Onco Targets Ther 13: 4257-4270, 2020.

15. Yu C, Wang Y, Liu T, Sha K, Song Z, Zhao M and Wang X: The microRNA miR-3174 suppresses the expression of ADAM15 and inhibits the proliferation of patient-derived bladder cancer cells. Onco Targets Ther 13: 4157-4168, 2020.

16. Zhou J, Dai W and Song J: miR-1182 inhibits growth and mediates the chemosensitivity of bladder cancer by targeting hTERT. Biochem Biophys Res Commun 470: 445-452, 2016.

17. Hou XS, Han CQ and Zhang W: MiR-1182 inhibited metastasis and proliferation of ovarian cancer by targeting hTERT. Eur Rev Med Pharmacol Sci 22: 1622-1628, 2018.

18. Zhang D, Xiao YF, Zhang JW, Xie R, Hu CJ, Tang B, Wang SM, Wu YY, Hao NB and Yang SM: miR-1182 attenuates gastric cancer proliferation and metastasis by targeting the open reading frame of hTERT. Cancer Lett 360: 151-159, 2015.

19. Huang C, Deng H, Wang Y, Jiang H, Xu R, Zhu X, Huang Z and Zhao X: Circular RNA circABCC4 as the ceRNA of miR-1182 facilitates prostate cancer progression by promoting FOXP4 expression. J Cell Mol Med 23: 6112-6119, 2019.

20. Dillon RL, White DE and Muller WJ: The phosphatidyl inositol 3-kinase signaling network: Implications for human breast cancer. Oncogene 26: 1338-1345, 2007.

21. Liby TA, Spyropoulos P, Buff Lindner H, Eldridge J, Beeson C, Hsu $\mathrm{T}$ and Muise-Helmericks RC: Akt3 controls vascular endothelial growth factor secretion and angiogenesis in ovarian cancer cells. Int J Cancer 130: 532-543, 2012.

22. Nakatani K, Thompson DA, Barthel A, Sakaue H, Liu W, Weigel RJ and Roth RA: Up-regulation of Akt3 in estrogen receptor-deficient breast cancers and androgen-independent prostate cancer lines. J Biol Chem 274: 21528-21532, 1999.
23. Lin HP, Lin CY, Huo C, Jan YJ, Tseng JC, Jiang SS, Kuo YY, Chen SC, Wang CT, Chan TM, et al: AKT3 promotes prostate cancer proliferation cells through regulation of Akt, B-Raf, and TSC1/TSC2. Oncotarget 6: 27097-27112, 2015.

24. Zhang F and Wu Z: Significantly altered expression of miR-511-3p and its target AKT3 has negative prognostic value in human prostate cancer. Biochimie 140: 66-72, 2017.

25. Lin HP, Jiang SS and Chuu CP: Caffeic acid phenethyl ester causes p21 induction, Akt signaling reduction, and growth inhibition in PC-3 human prostate cancer cells. PLoS One 7: e31286, 2012.

26. Wang G, Chen H and Liu J: The long noncoding RNA LINC01207 promotes proliferation of lung adenocarcinoma. Am J Cancer Res 5: 3162-3173, 2015.

27. Wang M, Lu X, Dong X, Hao F, Liu Z, Ni G and Chen D: pERK1/2 silencing sensitizes pancreatic cancer BXPC-3 cell to gemcitabine-induced apoptosis via regulating Bax and Bcl-2 expression. World J Surg Oncol 13: 66, 2015.

28. Wang Y, Li Z, Zheng S, Zhou Y, Zhao L, Ye H, Zhao X, Gao W, Fu Z, Zhou Q, et al: Expression profile of long non-coding RNAs in pancreatic cancer and their clinical significance as biomarkers. Oncotarget 6: 35684-35698, 2015.

29. Liu C, Wang JO, Zhou WY, Chang XY, Zhang MM, Zhang Y and Yang XH: Long non-coding RNA LINC01207 silencing suppresses AGR2 expression to facilitate autophagy and apoptosis of pancreatic cancer cells by sponging miR-143-5p. Mol Cell Endocrinol 493: 110424, 2019.

30. Livak KJ and Schmittgen TD: Analysis of relative gene expression data using real-time quantitative PCR and the 2(-Delta Delta C(T)) method. Methods 25: 402-408, 2002.

31. National Research Council (US) Committee for the Update of the Guide for the Care and Use of Laboratory Animals: Guide for the Care and Use of Laboratory Animals, 8th edition. https:/grants.nih.gov/grants/olaw/guide-for-the-care-and-useof-laboratory-animals.pdf. Accessed October 21, 2021.

32. Deng T, Yuan Y, Zhang C, Zhang C, Yao W, Wang C, Liu R and $\mathrm{Ba}$ Y: Identification of circulating miR-25 as a potential biomarker for pancreatic cancer diagnosis. Cell Physiol Biochem 39: 1716-1722, 2016

33. Cai B, Song XQ, Cai JP and Zhang S: HOTAIR: A cancer-related long non-coding RNA. Neoplasma 61: 379-391, 2014.

34. Pang EJ, Yang R, Fu XB and Liu YF: Overexpression of long non-coding RNA MALAT1 is correlated with clinical progression and unfavorable prognosis in pancreatic cancer. Tumor Biol 36: 2403-2407, 2015.

35. Wang S, Qiu J, Wang L, Wu Z, Zhang X, Li Q and Jiang F: Long non-coding RNA LINC01207 promotes prostate cancer progression by downregulating microRNA-1972 and upregulating LIM and SH3 protein 1. IUBMB Life 72: 1960-1975, 2020.

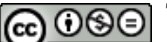

This work is licensed under a Creative Commons Attribution-NonCommercial-NoDerivatives 4.0 International (CC BY-NC-ND 4.0) License. 